\title{
Reproducibility of quantitative flow ratio: An inter-core laboratory variability study
}

\author{
Yunxiao Chang ${ }^{1 *}$, Liwei Chen ${ }^{2 *}$, Jelmer Westra ${ }^{3}$, Zhongwei Sun ${ }^{4}$, \\ Changdong Guan ${ }^{4}$, Yimin Zhang ${ }^{1}$, Daixin Ding ${ }^{1}$, Bo Xu ${ }^{4}$, Shengxian $\mathrm{Tu}^{1}$ \\ ${ }^{1}$ Biomedical Instrument Institute, School of Biomedical Engineering, \\ Shanghai Jiao Tong University, Shanghai, China \\ ${ }^{2}$ Department of Cardiology, Shin Kong Memorial Wu Ho-Su Hospital, Taipei, Taiwan \\ ${ }^{3}$ Department of Cardiology, Aarhus University Hospital, Skejby, Denmark \\ ${ }^{4}$ Fu Wai Hospital, National Center for Cardiovascular Diseases, \\ Chinese Academy of Medical Sciences, Beijing, China
}

\begin{abstract}
Background: Quantitative flow ratio (QFR) is a novel approach to derive fractional flow reserve (FFR) from coronary angiography. This study sought to evaluate the reproducibility of QFR when analyzed in independent core laboratories.

Methods: All interrogated vessels in the FAVOR II China Study were separately analyzed using the AngioPlus system (Pulse medical imaging technology, Shanghai) by two independent core laboratories, following the same standard operation procedures. The analysts were blinded to the FFR values and online QFR values. For each interrogated vessel, two identical angiographic image runs were used by two core laboratories for QFR computation. In both core laboratories QFR was successfully obtained in 330 of 332 vessels, in which FFR was available in 328 vessels. Thus, 328 vessels ended in the present statistical analysis.
\end{abstract}

Results: The mean difference in contrast-flow QFR between the two core laboratories was $0.004 \pm$ $\pm 0.03(p=0.040)$, which was slightly smaller than that between the online analysis and the two core laboratories $(0.01 \pm 0.05, p<0.001$ and $0.01 \pm 0.05, p=0.038)$. The mean difference of $Q F R$ with $r e-$ spect to FFR were comparable between the two core laboratories $(0.002 \pm 0.06, p=0.609$, and $0.002 \pm$ $\pm 0.06, p=0.531$ ). Receiver operating characteristic curve analysis showed that diagnostic accuracies of QFR analyzed by the two core laboratories were both excellent (area under the curve: $0.970 \mathrm{vs.} 0.963$, $p=0.142)$, when using FFR as the reference standard.

Conclusions: The present study showed good inter-core laboratory reproducibility of QFR in assessing functionally-significant stenosis. It suggests that QFR analyses can be carried out in different core laboratories if, and only if, highly standardized conditions are maintained. (Cardiol J 2020; 27, 3: 230-237)

Key words: quantitative flow ratio, fractional flow reserve, reproducibility, core laboratories, coronary stenosis

\section{Introduction}

Revascularization strategies of stable coronary artery disease (CAD) have relied largely on noninvasive stress tests and coronary angiography in current practice [1]. For decades physiologic evaluation of myocardial perfusion has been proposed and experimented with as a potential tool to determine the ischemic severity of coronary artery stenosis. However, it was not until recent

Address for correspondence: Shengxian Tu, PhD, FACC, FESC, Med-X Research Institute, Shanghai Jiao Tong University, No. 1954, Huashan Road, Xuhui District, Shanghai, 200030, China, tel: +86-21-6293-2631, fax: +86-21-6293-2156, e-mail: sxtu@sjtu.edu.cn

Received: 15.08.2018 Accepted: 16.08.2018

*The first two authors equally contributed. 
years, after the invention of clinically feasible tools, that physiological assessment of coronary blood flow could be adopted to guide interventions. Coronary fractional flow reserve (FFR) acquired by a pressure-wire placed within target coronary vessels can assist in decision making for intermediate lesions during coronary intervention, with proven clinical and economic benefits [2-5]. A FFR value of $\leq 0.80$ indicates a functionally significant stenosis [6]. The European Society of Cardiology has recommended this procedure as class I level A for the CAD revascularization approach [7]. Nevertheless, widespread utilization of FFR is limited by its invasive nature, relatively high cost and use of medication to achieve hyperemia states which may introduce complications or adverse events. A real-world survey reveals that FFR is used in less than $10 \%$ of intermediate coronary lesions (40-70\% stenosis) [8].

Quantitative flow ratio (QFR) is a novel angiography-based computation to derive FFR using computer algorithms that omits the need for pressure guidewire and vasodilator administration $[9,10]$. The entire analysis can be done in minutes without extra procedures during diagnostic coronary angiography, making it an appealing tool to be used in the catheterization laboratory. The recently published FAVOR II China Study demonstrated excellent accuracy of online QFR assessment in the catheterization laboratories when compared with the standard FFR measurement. Patient-level and vessel-level diagnostic accuracy of contrastflow QFR were $92.4 \%$ (95\% confidence interval [CI] 88.9-95.1\%) and 92.7\% (95\% CI 89.3-95.3\%), respectively [11].

In addition to the potential application in guiding coronary revascularization, QFR can be used to assess the efficacy of different stents by evaluating the physiological functionality of the coronary artery after stent implantation in a core laboratory setting [12]. The FAVOR II China Study also demonstrated that QFR showed excellent accuracy when analyzed in the core laboratory [11]. Nevertheless, the reproducibility of QFR when analyzed in other core laboratories has not been addressed. The aim of the present study was to evaluate the reproducibility of QFR when analyzed offline in two independent core laboratories.

\section{Methods}

\section{Study materials}

The FAVOR II China Study [11] is a prospective, multicenter study that enrolled patients who had at least one lesion with a diameter stenosis of $30 \%$ to $90 \%$ and a reference diameter $\geq 2 \mathrm{~mm}$ by visual estimation. Detailed inclusion and exclusion criteria of the study and the patient characteristics have been reported in the main study. All patients enrolled in the study were reanalyzed in a second core lab (CardHemo, Med-X Research Institute, Shanghai Jiao Tong University, Shanghai, China), hereafter noted as CoreLab2. The reference FFR values and the $Q F R$ values by the first core lab (CCRF, Beijing, China), hereafter noted as CoreLab1, were taken from the main study [11] to assess the inter-core laboratories variability and the difference in diagnostic accuracy by different core laboratories, when using FFR as the reference standard. The study procedure was approved by the institutional review board. All patients provided written informed consent.

\section{QFR core-lab analysis}

All QFR analyses in the CoreLab2 were performed by an experienced analyst (CY), following the same standard operation procedures (SOP) and by the AngioPlus system (Pulse medical imaging technology, Shanghai) as used by the CoreLab1. The analyst was blinded to the FFR values and the previously computed QFR values. For each interrogated vessel, the two identical angiographic image runs were used by the two core laboratories for QFR computation. The interrogated vessels were reconstructed based on two angiographic image runs with minimal overlap and foreshortening that were acquired with $\geq 25^{\circ}$ difference in projection angles. Subsequently, the analyst performed modified frame count on one of the angiographic runs to obtain the mean contrast flow velocity, from which the computer modeled the hyperemic flow velocity and computed the contrast-flow QFR for the entire vessel. This methodology has been previously reported $[9,10]$. In the same procedure, the computer used a fixed contrast flow velocity to derive the fixed-flow QFR (fQFR) [10]. Prior to QFR computation, the interrogated vessels and the segments where FFR was measured and reported to the analyst. However, the image frames used for three-dimensional (3D) angiographic reconstruction and the image runs used for modified frame count were selected by the analyst following SOP, being blinded to the online and the CoreLab1 selection results. Contour of the vessel lumen was first automatically delineated by the QFR measurement system and manual adjustment was allowed following SOP in case of suboptimal angiographic image quality. 


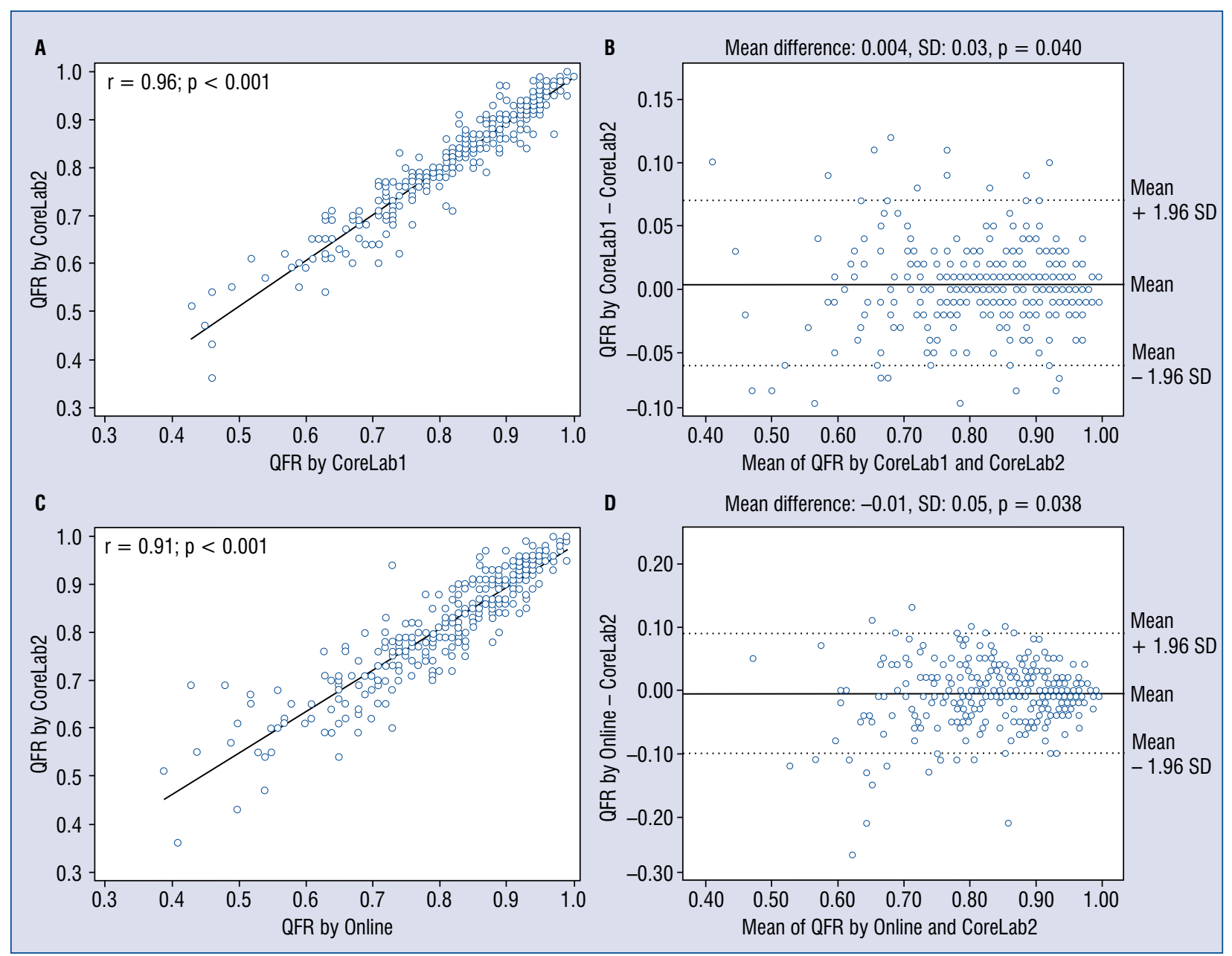

Figure 1. A-D. Correlation and agreement between quantitative flow ratio (QFR) analysis of CoreLab1 and CoreLab2, Online and CoreLab2; SD — standard deviation.

\section{Statistical analysis}

Continuous variables were depicted as mean \pm standard deviation unless otherwise stated. The D'Agostino Pearson test was used to test normal distribution of the data. Pair-wise comparisons of different QFR analyses were assessed using BlandAltman plots. The correlation between FFR and QFR was evaluated using the Pearson or Spearman correlation tests as appropriate. An FFR test $\leq 0.80$ was considered for diagnosis of hemodynamically-significance. Sensitivity, specificity, positive predictive value (PPV), negative predictive value (NPV), positive likelihood ratio (+LR), negative likelihood ratio (-LR) of QFR with the FFR as the reference standard were calculated and the 95\% CIs were added, as appropriate. The Student t test or Mann-Whitney U tests were performed for pairwise comparisons. Receiver operator characteristic (ROC) curves were compared using the DeLong method. All statistical analyses were performed using MedCalc 14.12.0 (MedCalc Software, Mariakerke, Belgium). A two-sided $\mathrm{p}$ value of $<0.05$ was considered statistically significant.

\section{Results}

In both core laboratories QFR and $\mathrm{fQFR}$ were successfully obtained in 330 of 332 interrogated vessels, in which FFR was available in 328 vessels from 304 patients. Thus, pairwise comparisons were performed in 328 vessels.

Correlation and agreement of QFR analysis

The computed QFR values by the two core laboratories demonstrated better correlations ( $\mathrm{r}=0.96$ vs. $0.91, \mathrm{p}<0.001)$ and agreement $(0.004 \pm$ \pm 0.03 vs. $-0.01 \pm 0.05, \mathrm{p}<0.001)$ than those by CoreLab2 and online analyses (Fig. 1). Similarly, computed fQFR by the two core laboratories had better correlations ( $r=0.93$ vs. $0.88, \mathrm{p}<0.001$ ) 


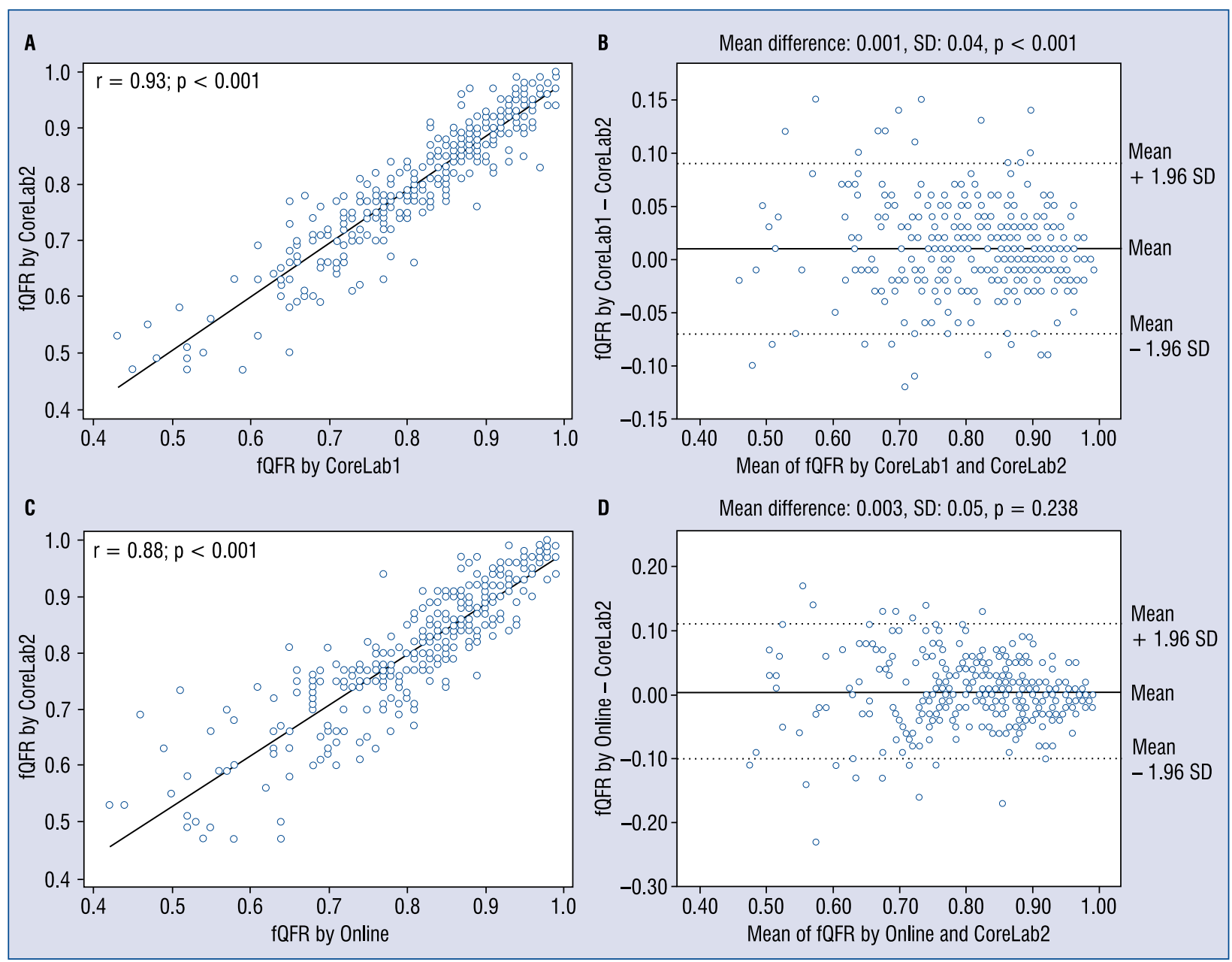

Figure 2. A-D. Correlation and agreement between fixed-flow quantitative flow ratio (fOFR) analysis of CoreLab1 and CoreLab2, Online and CoreLab2; SD — standard deviation.

and agreement $(0.01 \pm 0.04$ vs. $0.003 \pm 0.05$, $\mathrm{p}<0.001)$ than those by CoreLab2 and online analyses (Fig. 2). The computed QFR values by the two core laboratories demonstrated better correlations ( $\mathrm{r}=0.96$ vs. $0.91, \mathrm{p}<0.001)$ and agreement $(0.004 \pm$ \pm 0.03 vs. $-0.01 \pm 0.05, \mathrm{p}<0.001)$ than those by CoreLab1 and the online analyses reported in the FAVOR II China Study [11].

\section{Correlation and agreement}

between FFR and QFR analysis

For CoreLab2 analyses, both QFR and fQFR had good correlations with FFR, with the Spearman correlation coefficients being significantly higher for QFR than fQFR measurements (0.86 vs. 0.79, $\mathrm{p}<0.001)$. Both QFR and fQFR had good agreement with FFR, with a slightly smaller error for QFR than fQFR $(0.002 \pm 0.06$, vs. $0.01 \pm 0.08$, $\mathrm{p}<0.001$ ) (Fig. 3). The mean difference of QFR with respect to FFR were comparable between the two core laboratories $(0.0016 \pm 0.06$ vs. $0.0021 \pm$ $\pm 0.06, \mathrm{p}=0.915)$.

\section{Diagnostic performance of QFR and $\mathrm{AQFR}$} using FFR as the reference standard

ROC analyses show that QFR analyzed by the two core laboratories had comparable diagnostic performance, with areas under curve (AUC) being 0.970 and $0.963(\mathrm{p}=0.142)$. The fQFR analyzed by the second core laboratory also had comparable but statistically significant AUC as the first core laboratory ( 0.950 vs. $0.935, \mathrm{p}=0.047$ ) (Fig. 4).

Using FFR as the reference standard, the overall accuracy, sensitivity and specificity in diagnosis of hemodynamically-significant stenosis were all excellent for CoreLab2 (accuracy: 92.07\% [95\% CI 89.13-95.01\%], sensitivity: $94.96 \%$ [95\% CI 89.3$-98.1 \%$ ], specificity: $89.95 \%$ [95\% CI 85.1-93.7\%]), and those were comparable with CoreLab1 (accuracy: $93.3 \%$ [95\% CI 90.0-95.7\%], sensitivity: 


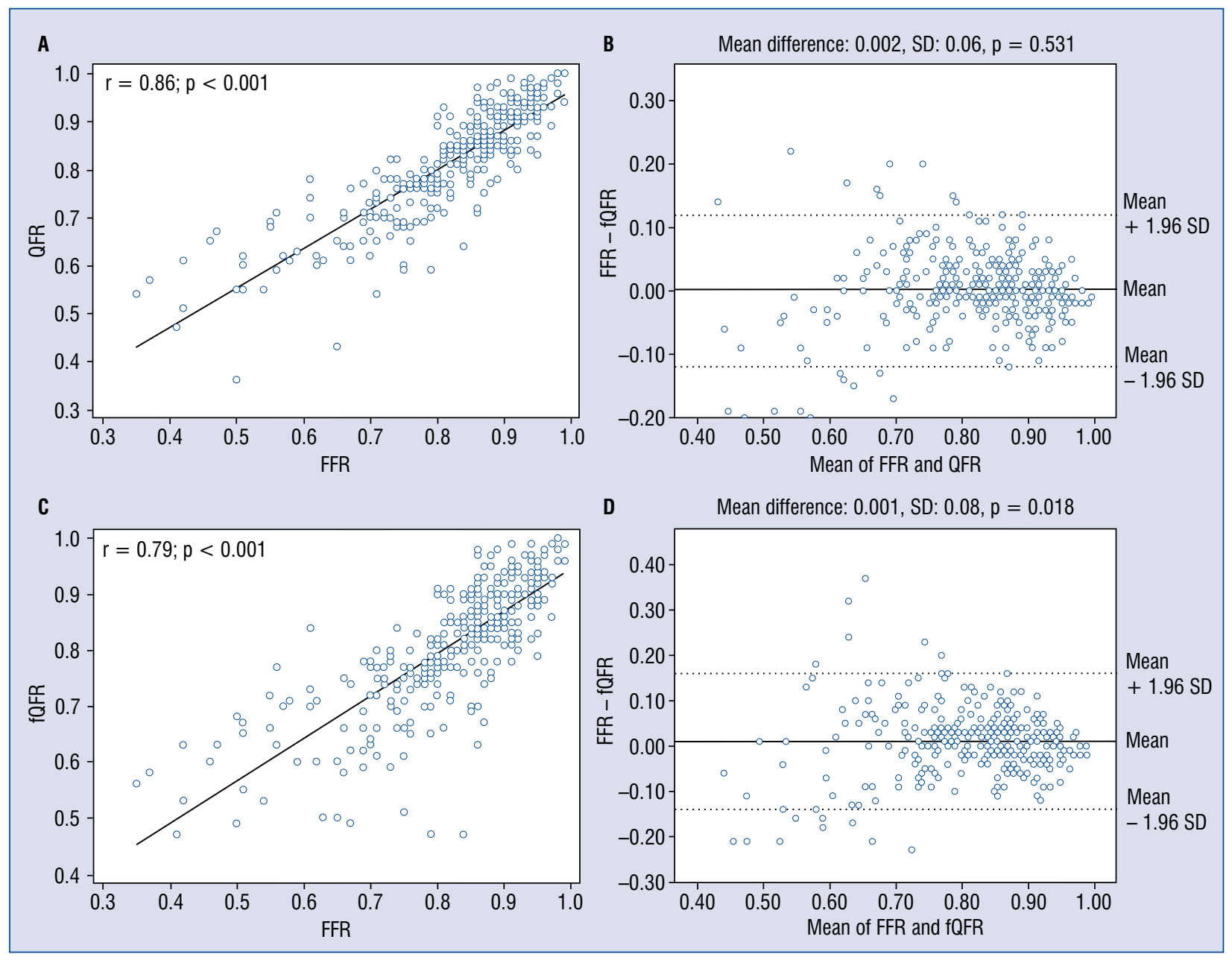

Figure 3. Correlation and agreement between fractional flow reserve (FFR) and quantitative flow ratio (QFR) analysis of CoreLab2 (A, B). Correlation and agreement between FFR and fixed-flow QFR analysis of CoreLab2 (C, D).
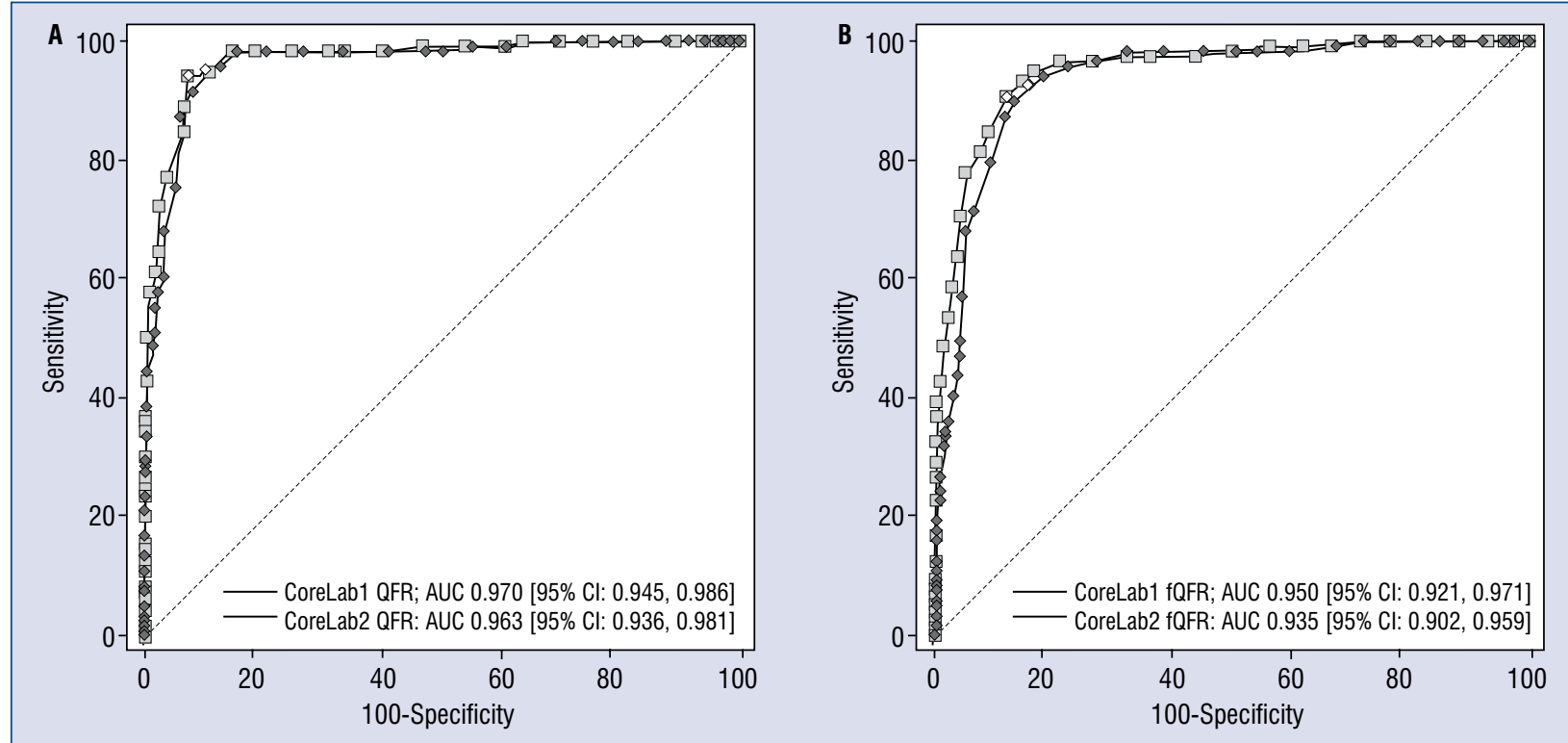

Figure 4. Comparison of receiver operating curves for the quantitative flow ratio (QFR) (A) and fixed-flow QFR (fQFR) (B) results of CoreLab1 and CoreLab2. QFR analyzed by the two core laboratories had comparable diagnostic performance. Fractional flow reserve was used as the reference standard; AUC - area under curve; $\mathrm{Cl}-\mathrm{confidence}$ interval. 
94.1\% [95\% CI 88.3-97.6\%], specificity: 92.8\% [95\% CI 88.4-95.9\%]) as previously reported [11]. PPV, NPV, +LR and -LR for CoreLab2 was 84.3, $96.9,9.45$ and 0.056 , respectively.

\section{Discussion}

This is the first study to report on the intercorelab variation for QFR computation. The key finding was that $\mathrm{QFR}$ is reproducible and provides comparable diagnostic accuracy with FFR as a reference standard when analyzed by two independent core laboratories using the same dedicated SOP. It supports the use of QFR for evaluation of coronary physiology in a core laboratory setting.

The study focused on the technical and observer-dependent variation since user-interactions are needed to refine geometrical parameters of vessels and select an appropriate frame for contrast flow velocity assessment. It showed herein, that very similar QFR results could be acquired in different core laboratories when the analysts strictly followed SOP and that the extra frame-counting step needed to compute QFR compared to $\mathrm{F} Q \mathrm{FR}$ did not influence reproducibility.

Fractional flow reserve has emerged as the current gold standard to guide percutaneous coronary interventions by improving outcome compared to angiography-guided percutaneous coronary intervention in multiple clinical trials $[6,7,13,14]$. Apart from the favorable clinical outcomes, FFR appears solid in terms of reproducibility. The absolute difference and standard deviation of repeated FFR measurements approaches $0.03 \pm$ \pm 0.02 as illustrated in the Fractional Flow Reserve to Determine the Appropriateness of Angioplasty in Moderate Coronary Stenosis A Randomized Trial (DEFER) where repeated FFR measurements were performed in 325 patients [15]. Application of the "smart minimum" algorithm to The Verification of Instantaneous Wave-Free Ratio and Fractional Flow Reserve for the Assessment of Coronary Artery Stenosis in Everyday Practice (VERIFY) study likewise found good repeatability of FFR $(0.00 \pm 0.02)$ [16]. The minor variability may be caused by several factors related to fluctuating hemodynamics, suboptimal vasodilation, drift and variation of wire-position. The presented inter-corelab variation for QFR of $0.00 \pm 0.03$ is in the same range as FFR and adds to the existing knowledge with multiple off-line and on-line studies proving high diagnostic accuracy of QFR when FFR is used as a reference standard [9-11, 17-23].
The present results confirm findings from a pilot study conducted by van Rosendael et al. [24] that presented an inter-observer variation of $0.02 \pm$ \pm 0.04 for QFR in a limited number of 20 lesions. Importantly, the present results are improved when compared to QFR intra-observer agreement of $0.00 \pm 0.06$ in 40 lesions, as recently reported by Westra et al. [19]. This is most likely due to a more refined SOP for image acquisition, better angiographic quality and a more elaborate protocol for QFR analysis. Further, results were marginally better than on-line QFR results in the FAVOR II China Study [11], which may be explained by multiple factors. In the on-line analyses, five centres participated in FAVOR II China Study [11] with their own technicians performing all measurements. Although highly skilled, inter-personnel differences may have contributed to slight variations. Further, time to QFR was recorded in the FAVOR II China Study [11]. Although this was not used for comparison, site staff facing time pressure to execute the measurements may have resulted in a minor bias.

\section{Limitations of the study}

This study has certain limitations. Firstly, intra-observer variation was not assessed. Secondly, despite the presented comparison of core-lab specific diagnostic accuracy estimates with FFR, it should be noted that this study does not report on true repeated QFR measurements since two independent core-labs used the same datasets to compute QFR at two different time-points. Hence, the biological variability of repeated QFR computation was not assessed. However, the majority of data on repeated FFR derives from repeated FFR measurements in the same patient within a small time-frame which likewise limits the evaluation of biological variability. Thirdly, optimal angiographic quality is crucial for optimal QFR computation. Since the repeated measurements were all performed on the same high-quality angiographic runs, the variation caused by potential suboptimal image quality was not included.

\section{Conclusions}

The present study showed good inter-core laboratory reproducibility for QFR in assessing the physiological significance of coronary stenosis. It suggests that QFR analyses can be carried out in different core laboratories if highly standardized operating procedures are maintained. 


\section{Funding}

This study was supported by the National Key Research and Development Program of China (Grant No. 2016YFC0100500), the Natural Science Foundation of China (Grant No. 31500797), Shanghai ShenKang Hospital Development Center (16CR3035A), and Shanghai Jiao Tong University (YG2015ZD04).

Conflict of interest: None of the Authors have declared a conflict of interest in relation to this study, with the exception that $\mathrm{S}$ Tu received research support from Medis medical imaging and Pulse medical imaging. Other authors report no conflicts of interest regarding this manuscript.

\section{References}

1. Wijns W, Kolh P, Danchin N. Guidelines on myocardial revascularization. Eur Heart J. 2010; 31: 2501-2555.

2. Corcoran D, Hennigan B, Berry C. Fractional flow reserve: a clinical perspective. Int J Cardiovasc Imaging. 2017; 33(7): 961-974, doi: 10.1007/s10554-017-1159-2, indexed in Pubmed: 28577046.

3. Tu S, Echavarria-Pinto M, von Birgelen C, et al. Fractional flow reserve and coronary bifurcation anatomy: a novel quantitative model to assess and report the stenosis severity of bifurcation lesions. JACC Cardiovasc Interv. 2015; 8(4): 564-574, doi: 10.1016/j.jcin.2014.12.232, indexed in Pubmed: 25819180.

4. Johnson NP, Tóth GG, Lai D, et al. Prognostic value of fractional flow reserve: linking physiologic severity to clinical outcomes. J Am Coll Cardiol. 2014; 64(16): 1641-1654, doi: 10.1016/j. jacc.2014.07.973, indexed in Pubmed: 25323250.

5. Fearon WF. Percutaneous coronary intervention should be guided by fractional flow reserve measurement. Circulation. 2014; 129(18): 1860-1870, doi: 10.1161/CIRCULATIONAHA.113.004300, indexed in Pubmed: 24799502.

6. De Bruyne B, Pijls NHJ, Kalesan B, et al. FAME 2 Trial Investigators. Fractional flow reserve-guided PCI versus medical therapy in stable coronary disease. N Engl J Med. 2012; 367(11): 991-1001, doi: 10.1056/NEJMoa1205361, indexed in Pubmed: 22924638.

7. Kolh P, Windecker S, Alfonso F, et al. 2014 ESC/EACTS Guidelines on myocardial revascularization: the Task Force on Myocardial Revascularization of the European Society of Cardiology (ESC) and the European Association for Cardio-Thoracic Surgery (EACTS). Developed with the special contribution of the European Association of Percutaneous Cardiovascular Interventions (EAPCI). Eur J Cardiothorac Surg. 2014; 46(4): 517-592, doi: 10.1093/ejcts/ezu366, indexed in Pubmed: 25173601.

8. Götberg M, Cook CM, Sen S, et al. The Evolving Future of Instantaneous Wave-Free Ratio and Fractional Flow Reserve. J Am Coll Cardiol. 2017; 70(11): 1379-1402, doi: 10.1016/j. jacc.2017.07.770, indexed in Pubmed: 28882237.

9. Tu S, Barbato E, Köszegi Z, et al. Fractional flow reserve calculation from 3-dimensional quantitative coronary angiography and TIMI frame count: a fast computer model to quantify the functional significance of moderately obstructed coronary arteries. JACC Cardiovasc Interv. 2014; 7(7): 768-777, doi: 10.1016/j. jcin.2014.03.004, indexed in Pubmed: 25060020.
10. Tu S, Westra J, Yang J, et al. FAVOR Pilot Trial Study Group. Diagnostic Accuracy of Fast Computational Approaches to Derive Fractional Flow Reserve From Diagnostic Coronary Angiography: The International Multicenter FAVOR Pilot Study. JACC Cardiovasc Interv. 2016; 9(19): 2024-2035, doi: 10.1016/j. jcin.2016.07.013, indexed in Pubmed: 27712739.

11. Xu Bo, Tu S, Qiao S, et al. Diagnostic accuracy of angiographybased quantitative flow ratio measurements for online assessment of coronary stenosis. J Am Coll Cardiol. 2017; 70(25): 3077-3087, doi: 10.1016/j.jacc.2017.10.035, indexed in Pubmed: 29101020.

12. Asano T, Katagiri Y, Collet C, et al. Functional comparison between the BuMA Supreme biodegradable polymer sirolimuseluting stent and a durable polymer zotarolimus-eluting coronary stent using quantitative flow ratio: PIONEER QFR substudy. EuroIntervention. 2018; 14(5): e570-e579, doi: 10.4244/EIJD-17-00461, indexed in Pubmed: 28994655.

13. Pijls NHJ, Fearon WF, Tonino PAL, et al. FAME Study Investigators. Fractional flow reserve versus angiography for guiding percutaneous coronary intervention. N Engl J Med. 2009; 360(3): 213224, doi: 10.1056/NEJMoa0807611, indexed in Pubmed: 19144937.

14. Pijls NHJ, Fearon WF, Tonino PAL, et al. FAME Study Investigators. Fractional flow reserve versus angiography for guiding percutaneous coronary intervention in patients with multivessel coronary artery disease: 2-year follow-up of the FAME (Fractional Flow Reserve Versus Angiography for Multivessel Evaluation) study. J Am Coll Cardiol. 2010; 56(3): 177-184, doi: 10.1016/j. jacc.2010.04.012, indexed in Pubmed: 20537493.

15. Pijls NHJ, van Schaardenburgh P, Manoharan G, et al. Percutaneous coronary intervention of functionally nonsignificant stenosis: 5-year follow-up of the DEFER Study. J Am Coll Cardiol. 2007; 49(21): 2105-2111, doi: 10.1016/j.jacc.2007.01.087, indexed in Pubmed: 17531660.

16. Johnson NP, Johnson DT, Kirkeeide RL, et al. Repeatability of Fractional Flow Reserve Despite Variations in Systemic and Coronary Hemodynamics. JACC Cardiovasc Interv. 2015; 8(8): 1018-1027, doi: 10.1016/j.jcin.2015.01.039, indexed in Pubmed: 26205441.

17. Yazaki K, Otsuka M, Kataoka S, et al. Applicability of 3-dimensional quantitative coronary angiography-derived computed fractional flow reserve for intermediate coronary stenosis. Circ J. 2017; 81(7): 988-992, doi: 10.1253/circj.CJ-16-1261, indexed in Pubmed: 28331135.

18. Smit JM, Koning G, van Rosendael AR, et al. Relationship between coronary contrast-flow quantitative flow ratio and myocardial ischemia assessed by SPECT MPI. Eur J Nucl Med Mol Imaging. 2017; 44(11): 1888-1896, doi: 10.1007/s00259-017-3769-2, indexed in Pubmed: 28685228.

19. Westra J, Tu S, Winther S, et al. Evaluation of coronary artery stenosis by quantitative flow ratio during invasive coronary angiography: the WIFI II study (wire-free functional imaging II). Circ Cardiovasc Imaging. 2018; 11(3): e007107, doi: 10.1161/ CIRCIMAGING.117.007107, indexed in Pubmed: 29555835.

20. Nam CW, Mangiacapra F, Entjes R, et al. FAME Study Investigators. Functional SYNTAX score for risk assessment in multivessel coronary artery disease. J Am Coll Cardiol. 2011; 58(12): 1211-1218, doi: 10.1016/j.jacc.2011.06.020, indexed in Pubmed: 21903052.

21. Spitaleri G, Tebaldi M, Biscaglia S, et al. Quantitative Flow Ratio Identifies Nonculprit Coronary Lesions Requiring Revascularization in Patients With ST-Segment-Elevation Myocardial Infarction and Multivessel Disease. Circ Cardiovasc 
Interv. 2018; 11(2): e006023, doi: 10.1161/CIRCINTERVENTIONS.117.006023, indexed in Pubmed: 29449325.

22. Emori H, Kubo T, Kameyama T, et al. Diagnostic accuracy of quantitative flow ratio for assessing myocardial ischemia in prior myocardial infarction. Circ J. 2018; 82(3): 807-814, doi: 10.1253/ circj.CJ-17-0949, indexed in Pubmed: 29343675.

23. Mejía-Rentería $\mathrm{H}$, Lee JM, Lauri F, et al. Influence of microcirculatory dysfunction on angiography-based functional as- sessment of coronary stenoses. JACC Cardiovasc Interv. 2018; 11(8): 741-753, doi: 10.1016/j.jcin.2018.02.014, indexed in Pubmed: 29673505 .

24. van Rosendael AR, Koning G, Dimitriu-Leen AC, et al. Accuracy and reproducibility of fast fractional flow reserve computation from invasive coronary angiography. Int J Cardiovasc Imaging. 2017; 33(9): 1305-1312, doi: 10.1007/s10554-017-1190-3, indexed in Pubmed: 28642995. 